\title{
Molecular characterization of a defensin gene from a hard tick, Dermacentor silvarum
}

\author{
Juanjuan Wang ${ }^{1}$, Gang Bian', Wen Pan ${ }^{1}$, Tingting Feng ${ }^{1,3^{*}}$ and Jianfeng Dai ${ }^{1,2,3^{*}}$
}

\begin{abstract}
Background: Ticks are distributed worldwide and considered as vectors of many human diseases. Tick defensins, a family of antimicrobial peptides, form the first line of defense against pathogens.

Findings: A defensin-like gene, named Ds-defensin, was identified from a cDNA library of the hard tick Dermacentor silvarum collected from northeast China. The full-length cDNA of Ds-defensin was 225 bp, encoding a 74 amino acid peptide. The nucleotide sequence of Ds-defensin shared 98.2\% similarity to putative defensin from Dermacentor marginatus. RT-PCR results suggested that Ds-defensin was extensively expressed in tick salivary gland and midgut, with a higher expression level in midgut. Ds-defensin showed broad antimicrobial activity against various Gram-positive and Gram-negative bacteria, as well as the fungus Candida albicans.

Conclusions: We characterized a functional defensin from D. silvarum of China. Ds-defensin showed bactericidal activity against various Gram-positive and Gram-negative bacteria. Ds-defensin can be expected to be introduced to the medical field as a new molecule with antibacterial activity.
\end{abstract}

Keywords: Defensin, Antimicrobial peptide (AMP), Tick, Dermacentor silvarum

\section{Findings}

Ticks, important medical arthropods, have great effects on animal and human health by transmitting various pathogens worldwide. Tick-borne pathogens include viruses, spirochetes, rickettsia, bacteria, and protozoa. They cause diseases such as tick-borne encephalitis, Crimean-Congo hemorrhagic fever, Lyme disease, Q fever, and Rocky Mountain spotted fever. In recent years, a new series of tick-borne diseases have posed a threat to the survival of mankind $[1,2]$. Many serological surveys also indicated the existence of populations with a variety of tick-borne infectious disease antibodies [3,4].

Hard ticks feed on mammals for a long time (several days), so that they have many opportunities to encounter microbes. Ticks do not have lymphocytes, thymuses, or antibodies. They rely heavily on antimicrobial peptides (AMPs) to defend against microbes so that they can live harmoniously with microbes $[5,6]$. AMPs are innate immune molecules that kill pathogenic microbes. Defensin

\footnotetext{
*Correspondence: ttfeng@suda.edu.cn; daijianfeng@suda.edu.cn ${ }^{1}$ Institute of Biology and Medical Sciences, Jiangsu Key Laboratory of Infection and Immunity, Soochow University, Suzhou City, Jiangsu Province, People's Republic of China

${ }^{3}$ Soochow University, Building 703, 199 Ren-ai Road, Suzhou 215123, P.R. China Full list of author information is available at the end of the article
}

is a well-known AMP in ticks. Defensins have been isolated from many species, including mammals, insects, and plants, and provide initial defense against infectious pathogens $[7,8]$. Several defensins and their isoforms have been identified in tick species including Ixodes scapularis, Amblyomma americanum, Dermacentor variabilis, Rhipicephalus microplus, Ornithodoros moubata, Ixodes ricinus, Amblyomma hebraeum and Haemaphysalis longicornis ticks [9-13]. Tick defensins usually contain six cysteine residues, and are usually expressed in the midgut (MG) after blood feeding or pathogen invasion $[14,15]$. Antimicrobial activity is primarily directed against Gram-positive bacteria, but some isoforms are also effective against Gram-negative bacteria, protozoa, and yeasts $[9,15-17]$.

In our study, we characterized a defensin gene from a cDNA library of Dermacentor silvarum. This hard tick species was collected in the field of Heilongjiang Province, located in northeast China. It has been reported to transmit human pathogens, such as tick-borne encephalitis virus, Anaplasma, and Rickettsia [18-20].

By sequencing a cDNA library of D. silvarum, a cDNA clone encoding the precursor of a putative defensin was obtained and named Ds-defensin. The cDNA sequence 
and deduced amino acid sequence of Ds-defensin are shown in Figure 1. Sequence analysis indicated that the Ds-defensin cDNA was 225 bp long, encoding a 74 amino acid peptide. The predicted protein contained a putative signal peptide cleavage site at amino acid positions 22 to 23, as analyzed by SignalP4.1 software (http://www.cbs.dtu.dk/services/SignalP/). The sequence of Ds-defensin cDNA has been deposited in GenBank with accession number KJ885301.

To identify the evolutionary relationships between Dsdefensin and other defensin-like genes in invertebrate species, sequence alignment and phylogenetic analysis were conducted using multiple sequence alignment software Clustal X2 (http://www.clustal.org/). The nucleotide sequence of Ds-defensin shared $98.2 \%$ identity with a putative defensin from Dermacentor marginatus, and their amino acid sequences were $100 \%$ identical, suggesting that these two tick species were closely related (Figure 2). Sequence alignment results suggest that Ds-defensin shared high similarity to putative defensins from hard tick species, such as $R$. microplus (79.73\%), I. ovatus (58.11\%), I. persulcatus (59.46\%), and A. americanum (55.56\%), and low similarity to putative defensins from soft ticks, such as O. moubata (43.84\%), O. papillipes (43.84\%), O. rostratus (43.84\%), and C. puertoricensis (43.84\%). All these defensins contained six conserved cysteine residues, including defensins from fruit fly (Drosophila melanogaster) or marine mollusk species (Crassostrea gigas and Ruditapes philippinarum) (Figure 2A). Phylogenetic tree analysis also suggested that these putative defensin sequences fell into three distinct clusters. One cluster comprised defensins from hard ticks (D. silvarum, D. marginatus, R. microplus, A. monolakensis, A. americanum, I. ovatus, and $I$. persulcatus). Another cluster comprised defensins from soft ticks (O. moubata, O. papillipes, O. rostratus, and C. puertoricensis), and the other cluster comprised defensins from marine mollusk species (A. americanum and $R$. philippinarum) (Figure 2B). Although defensins were evolutionarily conserved at the critical amino acid positions (six cysteine residues), they also exhibited significant changes during evolution.

1 atgcgcggactttgcatctgccttgtctttatccttgtctgtggt \begin{tabular}{lllllllllllllll}
$M$ & $R$ & $G$ & $L$ & $C$ & $I$ & $C$ & $L$ & $V$ & $F$ & I & L & V & $C$ & G \\
\hline
\end{tabular}

$46 \overline{\text { cttctaacegccacggeggecgtcceggctgaaagegaggeggct }}$ $\begin{array}{lllllllllllllll}\mathrm{L} & \mathrm{L} & \mathrm{T} & \mathrm{A} & \mathrm{T} & \mathrm{A} & \mathrm{A} & \mathrm{V} & \mathrm{P} & \mathrm{A} & \mathrm{E} & \mathrm{S} & \mathrm{E} & \mathrm{A} & \mathrm{A}\end{array}$

$91 \frac{\mathrm{L}}{\text { cacctcotgttcgtcgaggettcgggtgccccctgaaccaaggc }}$ $\begin{array}{lllllllllllllll}H & L & R & V & R & R & G & F & G & C & P & L & N & Q & G\end{array}$

136 gcctgtcacaaccactgccgcagcattcggegccgaggcggctac $\begin{array}{lllllllllllllllllllllll}\text { A } & C & H & N & H & C & R & S & I & R & R & R & G & G & Y\end{array}$

181 tgctctggcataatcaagcagacctgcacctgctacaggaattaa 225 $\begin{array}{llllllllllllllllllllll}C & S & G & I & I & K & Q & T & C & T & C & Y & R & N & *\end{array}$

Figure 1 Nucleotide and deduced amino acid sequences of Ds-defensin from Dermacentor silvarum. Putative signal peptide of Ds-defensin, as predicted by SignalP 4.1 server, is underlined.
To investigate the mRNA expression of Ds-defensin in different tissues of $D$. silvarum, RNA was extracted from whole ticks, salivary gland (SG), and midgut (MG). Transcript expression analysis of the Ds-defensin gene was conducted by RT-PCR using Ds-defensin genespecific primers. As shown in Figure 3, Ds-defensin was extensively expressed in MG and SG, and the expression level in MG was significantly higher than that in SG or whole ticks (Figure 3B). These data were consistent with previous findings, and suggest that Ds-defensin may play important roles in protecting ticks against microorganisms in MG and SG.

To test the antimicrobial activity of Ds-defensin, the mature form of Ds-defensin (a 52 amino acid peptide) was synthesized and purified by high-performance liquid chromatography (GL Biochem, Shanghai, China). The peptide was dissolved in PBS buffer containing 0.05\% Tween 20 and $1 \mu \mathrm{M} \beta$-merkaptoethanol (at a stock concentration of $1000 \mu \mathrm{M}$ ), and diluted properly when used in the antimicrobial assay. The target bacteria used in the bactericidal assay were purchased from China Veterinary Culture Collection Center (CVCC). Four Gram-positive bacteria, namely, Staphylococcus aureus (CMCC26003), Bacillus pumilus (CMCC63202), Micrococcus luteus (CMCC63202), and Mycobacterium bovis (carbenicillin-resistant strain); and three Gram-negative bacteria, namely, Salmonella typhimurium (CVCC542), Pseudomonas aeruginosa (CVCC2000), and Escherichia coli (CMCC44103), were used in this study. Microbial strains were grown to an $\mathrm{OD}_{600 \mathrm{~nm}}$ of $0.4-0.6$ at $37^{\circ} \mathrm{C}$ in Poor Broth media ( $1 \% \mathrm{w} / \mathrm{v}$ tryptone and $1 \% \mathrm{w} / \mathrm{v} \mathrm{NaCl}$ ) (except for M. bovis, which was grown in Middlebrook 7H9 Broth (BD-Difco, USA) with carbenicillin). Approximately $90 \mu \mathrm{L}$ of inocula of microbial strains (diluted with $\mathrm{PB}$ media to an $\mathrm{OD}_{600 \mathrm{~nm}}$ of 0.01 ) and $10 \mu \mathrm{L}$ of various concentrations of Ds-defensin $(0.1-50 \mu \mathrm{M})$ were added to the well of a 96-well plate. The mixture was grown for $20 \mathrm{~h}$ (for M. bovi, incubated for $48 \mathrm{~h}$ ) at $37^{\circ} \mathrm{C}$ and $250 \mathrm{rpm}$. Antimicrobial activity was evaluated by measuring the absorbance of the bacterial culture at $\mathrm{OD}_{600 \mathrm{~nm}}$. As shown in Table 1, Ds-defensin showed significant antimicrobial activities against Gram-positive bacteria with a minimal inhibitory concentration (MIC) less than $50 \mu \mathrm{M}$. For B. pumilus, Ds-defensin showed potent antimicrobial activities at $1 \mu \mathrm{M}$. Ds-defensin was less effective to Gram-negative bacteria. The MIC values to $S$. typhimurium and $P$. aeruginosa were greater than $50 \mu \mathrm{M}$. Ds-defensin did not inhibit the growth of E. coli, but it significantly inhibited the growth of antibioticresistant $M$. bovis $(\mathrm{MIC}=20 \mu \mathrm{M})$. To test whether Ds-defensin also influences the growth of fungi, Candida albicans (CAU0037) was used in the antimicrobial assay. C. albicans replication was significantly inhibited when the concentration of Ds-defensin exceeded $10 \mu \mathrm{M}$ in 


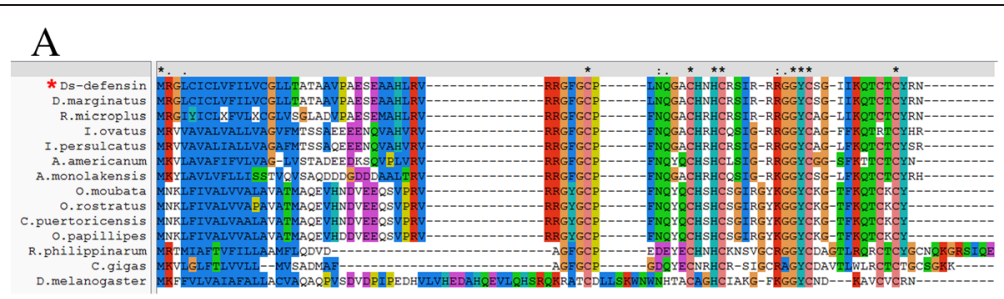

B

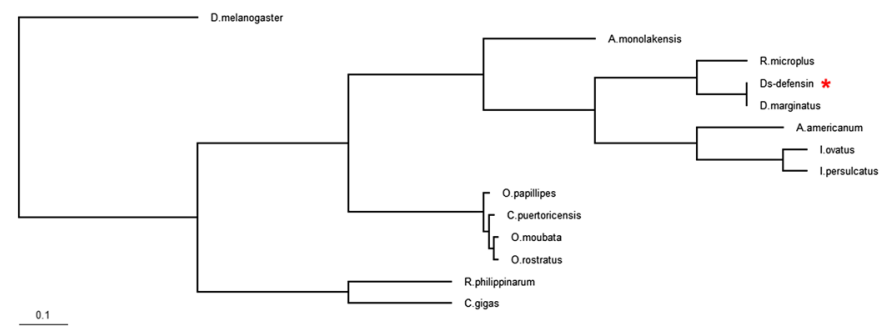

Figure 2 Sequence alignment (A) and phylogenetic tree (B) constructed with sequences of putative defensins from Dermacentor silvarum and other species. Ds-defensin is indicated with a red asterisk. Sequence GenBank accession numbers: Rhipicephalus microplus [AAO48943], Ornithodoros moubata [BAB41028], Ornithodoros papillipes [ACJ04425.1], Ornithodoros rostratus [ACJ04428.1], Carios puertoricensis [ACJ04429.1], Argas monolakensis [ABI52766.1], Dermacentor marginatus [ACJ04433.1], Ixodes ovatus [BAH09305.1], Ixodes persulcatus [BAH09304.1], Amblyomma americanum [ABI74752.1], Ruditapes philippinarum [ADO32580.1], Crassostrea gigas [ACQ76287.1], and Drosophila melanogaster [AAF58855.1].

Modified Martin Broth (Solarbio, Beijing, China), but the MIC to C. albicans was greater than $50 \mu \mathrm{M}$ (Table 1). As shown by hemolytic assay, Ds-defensin was harmless to human erythrocytes, in concentrations of up to $20 \mu \mathrm{M}$ (Additional file 1: Figure S1, A). Furthermore, Ds-defensin showed no detectable cytotoxicity to mammalian cell lines (Additional file 1: Figure S1, B), and did not infect the replication of vesicular stomatitis virus (VSV) in $293 \mathrm{~T}$ cells (data not shown).

Defensins are present in all types of organisms from humans and plants to arthropods. Defensins are AMPs that form the first line of defense against pathogens. A common function of defensins from all organisms is to lyse bacterial cells; however, the amino acid sequences of

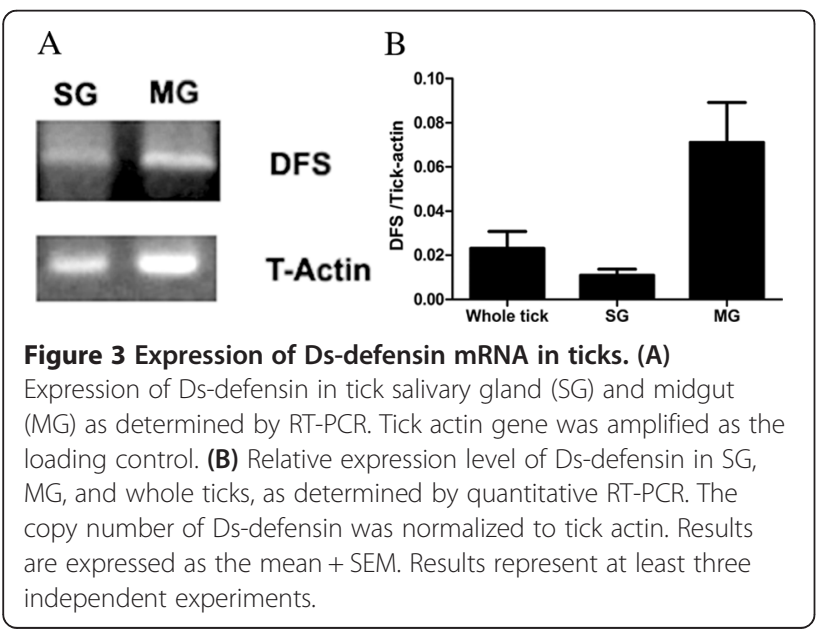

defensins show high diversity. Sequence analysis showed that the Ds-defensin peptide had $98.2 \%$ identity at the nucleotide level and $100 \%$ identity at the amino acid level to a putative defensin from $D$. marginatus, another hard tick species. Our data also show that defensins from hard and soft ticks shared a high degree of variability. The differentiation could be influenced by a diverse strategy of blood intake and types of pathogens that each tick species has encountered during their evolutionary history and geographical isolation [21].

The incidence of tick-borne diseases has steadily increased over the past few years, and effective vaccines

Table 1 Antimicrobial activity of Ds-defensin

Strain of microbes MIC $(\mu \mathrm{M})$

Gram-positive bacteria

Bacillus pumilus (CMCC63202)

Staphylococcus aureus (CMCC26003)

Micrococcus luteus (CMCC63202)

Mycobacterium bovis (carbenicillin-resistant)

20

\section{Gram-negative bacteria}

Salmonella typhimurium (CVCC542)

Pseudomonas aeruginosa (CVCC2000)

Escherichia coli (CMCC44103)

no effect

\section{Fungus}

Candida albicans (CAU0037)

$>50$

MIC: minimal peptide concentration required for total inhibition of cell growth in liquid medium. Determination of MICs was performed at least three times in triplicates. 
against most tick-borne pathogens are currently unavailable [22]. Defensin is an AMP that is not yet affected by antibiotic-resistance mechanisms [23]. Defensins have been identified in both soft and hard ticks, and they show different profiles of antimicrobial activity. For example, Chrudimská and colleagues characterized two defensin isoforms (Def1 and Def2) from the hard tick I. ricinus, and showed that both Def1 and Def2 have significant bactericidal effects on Gram-positive bacteria but they are insensitive to Gram-negative bacteria, yeasts, and viruses [24]. The same group recently characterized another defensin (defDM) from the hard tick $D$. marginatus, and suggested that defDM has inhibitory effects on Gram-positive bacteria and a popular tick-borne pathogen Borrelia afzelii [25]. Lu et al. reported that a defensin-like peptide (DLP) from hard tick H. longicornis has potent antimicrobial activities against bacteria and fungi (C. albicans), and even shows strong antimicrobial ability against drug-resistant microorganisms [6]. A recent review by Wang et al. summarized that the hard tick I. scapularis has two multigene families of DLPs [26]. The core region of $I$. scapularis defensin scapularisin-20 exhibits a wide spectrum of antimicrobial activity against both Gram-positive and Gram-negative bacteria, with higher potency to Gram-positive bacteria than to Gramnegative bacteria. The novel defensin we identified from the hard tick D. silvarum also has a distinct antimicrobial profile to different microbes. Ds-defensin showed strong potency against some Gram-positive bacteria ( $S$. aureus, B. pumilus, M. luteus, and M. bovis), but had less effect on Gram-negative bacteria (S. typhimurium, P. aeruginosa, and E. coli). At a dose higher than $10 \mu \mathrm{M}$, Ds-defensin showed significant antifungal activity against $C$. albicans growth and could inhibit the antibiotic-resistant strain of $M$. bovis. Ds-defensin was not hemolytic at $20 \mu \mathrm{M}$, and had no detectable cytotoxicity against various human cells. These data suggest that Ds-defensin could be safely used in mammalian systems as a potential antimicrobial reagent against various Gram-positive bacteria and some fungi.

\section{Additional file}

Additional file 1: Figure S1. Hemolytic and cytotoxic assay of Ds-defensin. (A) Hemolytic effect of Ds-defensin to mouse erythrocytes. Hemolytic activity was measured by co-incubating $2 \%$ (vol/vol) erythrocyte suspension with various concentration of Ds-defensin for 2 hours at $37^{\circ} \mathrm{C}$. (B) Ds-defensin had no cytotoxic effect on mammalian cell lines. Ds-defensin was added into the cell media at a final concentration of $10 \mu \mathrm{M}$ and incubated with various cells for $24 \mathrm{~h}$. The cell viabilities were measured using a CellTiter-Glo kit (Promega, USA).

\section{Authors' contributions}

TF and JD designed the experiments and prepared the manuscript. JW, GB, WP and TF performed all the experiments. All authors read and approved the final manuscript.

\section{Acknowledgements}

We thank Yikai Huang, Jiawei Tang, Tingting Li and Dan Cao for assistance with the experiments. This work was supported by A Project Funded by the Priority Academic Program Development of Jiangsu Higher Education Institutions; National Natural Science Foundation of China (NSFC) (81172812, 81271792, 31200648, 31300714, 81471571 and 31400737), PostDoc Science Foundation (2013 M541725) and Jiangsu Natural Science Foundation (BK2012180). J.D. is a member of Jiangsu Provincial Innovative Research Team.

\section{Author details}

${ }^{1}$ Institute of Biology and Medical Sciences, Jiangsu Key Laboratory of Infection and Immunity, Soochow University, Suzhou City, Jiangsu Province, People's Republic of China. ${ }^{2}$ Wuxi Medical School, Jiangnan University, Wuxi City, Jiangsu Province, People's Republic of China. ${ }^{3}$ Soochow University, Building 703, 199 Ren-ai Road, Suzhou 215123, P.R. China.

Received: 18 June 2014 Accepted: 21 November 2014

Published online: 15 January 2015

\section{References}

1. Vilcins IM, Old JM, Deane E. Molecular detection of Rickettsia, Coxiella and Rickettsiella DNA in three native Australian tick species. Exp Appl Acarol. 2009:49(3):229-42

2. Anderson JF, Magnarelli LA. Biology of ticks. Infect Dis Clin North Am. 2008;22(2):195-215. v.

3. Grzeszczuk A, Stanczak J, Kubica-Biernat B. Serological and molecular evidence of human granulocytic ehrlichiosis focus in the Bialowieza Primeval Forest (Puszcza Bialowieska), northeastern Poland. Eur J Clin Microbiol Infect Dis. 2002;21(1):6-11.

4. Zhan L, Cao WC, Chu CY, Jiang BG, Zhang F, Liu W, et al. Tick-borne agents in rodents, China, 2004-2006. Emerg Infect Dis. 2009;15(12):1904-8.

5. Zasloff M. Antimicrobial peptides of multicellular organisms. Nature. 2002;415(6870):389-95.

6. Lu X, Che Q, LV Y, Wang M, Lu Z, Feng F, et al. A novel defensin-like peptide from salivary glands of the hard tick, Haemaphysalis longicornis. Protein Sci. 2010;19(3):392-7.

7. Kopacek P, Hajdusek O, Buresova V, Daffre S. Tick innate immunity. Adv Exp Med Biol. 2010;708:137-62.

8. Telleria EL, Sant'Anna MR, Alkurbi MO, Pitaluga AN, Dillon RJ, Traub-Cseko YM. Bacterial feeding, Leishmania infection and distinct infection routes induce differential defensin expression in Lutzomyia longipalpis. Parasit Vectors. 2013;6:12.

9. Nakajima Y, Ishibashi J, Yukuhiro F, Asaoka A, Taylor D, Yamakawa M. Antibacterial activity and mechanism of action of tick defensin against Gram-positive bacteria. Biochim Biophys Acta. 2003;1624(1-3):125-30.

10. Lai R, Lomas LO, Jonczy J, Turner PC, Rees HH. Two novel non-cationic defensin-like antimicrobial peptides from haemolymph of the female tick, Amblyomma hebraeum. Biochem J. 2004;379(Pt 3):681-5.

11. Rudenko N, Golovchenko M, Grubhoffer L. Gene organization of a novel defensin of Ixodes ricinus: first annotation of an intron/exon structure in a hard tick defensin gene and first evidence of the occurrence of two isoforms of one member of the arthropod defensin family. Insect Mol Biol. 2007;16(4):501-7.

12. Rudenko N, Golovchenko M, Edwards MJ, Grubhoffer L. Differential expression of Ixodes ricinus tick genes induced by blood feeding or Borrelia burgdorferi infection. J Med Entomol. 2005;42(1):36-41.

13. Zhou J, Liao M, Ueda M, Gong H, Xuan X, Fujisaki K. Sequence characterization and expression patterns of two defensin-like antimicrobial peptides from the tick Haemaphysalis longicornis. Peptides. 2007;28(6):1304-10.

14. Kocan KM, de la Fuente J, Manzano-Roman R, Naranjo V, Hynes WL, Sonenshine DE. Silencing expression of the defensin, varisin, in male Dermacentor variabilis by RNA interference results in reduced Anaplasma marginale infections. Exp Appl Acarol. 2008;46(1-4):17-28.

\section{Competing interests}

The authors declare that they have no competing interests. 
15. Saito $Y$, Konnai S, Yamada S, Imamura S, Nishikado H, Ito T, et al. Identification and characterization of antimicrobial peptide, defensin, in the taiga tick, Ixodes persulcatus. Insect Mol Biol. 2009;18(4):531-9.

16. Tsuji N, Battsetseg B, Boldbaatar D, Miyoshi T, Xuan X, Oliver Jr JH, et al. Babesial vector tick defensin against Babesia sp. parasites. Infect Immun. 2007:75(7):3633-40.

17. Isogai E, Isogai H, Takahashi K, Kobayashi-Sakamoto M, Okumura K. Antimicrobial activity of three tick defensins and four mammalian cathelicidin-derived synthetic peptides against Lyme disease spirochetes and bacteria isolated from the midgut. Exp Appl Acarol. 2009;49(3):221-8.

18. Wen J, Jiao D, Wang JH, Yao DH, Liu ZX, Zhao G, et al. Rickettsia raoultii, the predominant Rickettsia found in Dermacentor silvarum ticks in China-Russia border areas. Exp Appl Acarol. 2014:63(4):579-85.

19. Zhang L, Liu H, Xu B, Lu Q, Li L, Chang L, et al. Anaplasma phagocytophilum infection in domestic animals in ten provinces/cities of China. Am J Trop Med Hyg. 2012;87(1):185-9.

20. Tian ZC, Liu GY, Shen H, Xie JR, Luo J, Tian MY. First report on the occurrence of Rickettsia slovaca and Rickettsia raoultii in Dermacentor silvarum in China. Parasit Vectors. 2012:5:19.

21. Chrudimska T, Chrudimsky T, Golovchenko M, Rudenko N, Grubhoffer L. New defensins from hard and soft ticks: similarities, differences, and phylogenetic analyses. Vet Parasitol. 2010;167(2-4):298-303.

22. Clark RP, Hu LT. Prevention of lyme disease and other tick-borne infections. Infect Dis Clin North Am. 2008:22(3):381-96. vii.

23. Hancock RE. Cationic peptides: effectors in innate immunity and novel antimicrobials. Lancet Infect Dis. 2001;1(3):156-64.

24. Chrudimska T, Slaninova J, Rudenko N, Ruzek D, Grubhoffer L. Functional characterization of two defensin isoforms of the hard tick /xodes ricinus. Parasit Vectors. 2011;4:63.

25. Chrudimska T, Cerovsky V, Slaninova J, Rego RO, Grubhoffer L. Defensin from the ornate sheep tick Dermacentor marginatus and its effect on Lyme borreliosis spirochetes. Dev Comp Immunol. 2014;46(2):165-70.

26. Wang $Y$, Zhu S. The defensin gene family expansion in the tick /xodes scapularis. Dev Comp Immunol. 2011;35(11):1128-34.

\section{Submit your next manuscript to BioMed Central and take full advantage of:}

- Convenient online submission

- Thorough peer review

- No space constraints or color figure charges

- Immediate publication on acceptance

- Inclusion in PubMed, CAS, Scopus and Google Scholar

- Research which is freely available for redistribution 